\title{
El diseño de material didáctico como aporte al abordaje de los problemas ambientales en entornos educativos y comunitarios
}

\section{Design of Educational Material as a Contribution to Environmental Problems in Educational and Community}

\author{
Julián David Becerra-Barón ${ }^{1}$ \\ Corporación Autónoma Regional de Boyacá CORPOBOYACÁ \\ Boyacá, Colombia \\ jdbbecerra@hotmail.com
}

Nidia Yaneth Torres-Merchán ${ }^{2}$

Facultad de Educación

Universidad Pedagógica y Tecnológica de Colombia

Boyacá, Colombia

nidia.torres@uptc.edu.co

Recibido: 12 Agosto 2012 Aceptado: 26 junio 2014 Corregido: 4 Julio 2014

Resumen. La necesidad de diseñar materiales que promuevan reflexiones alrededor de los problemas ambientales, hace que las instituciones educativas asuman escenarios promotores en la generación de estrategias didácticas en relación al tema. En este trabajo, se presenta el proceso de diseño de un módulo didáctico denominado: "Reflexionemos sobre los Problemas Ambientales del Municipio de Tunja”, que tiene como finalidad el reconocimiento de los problemas ambientales más relevantes que afectan a esta ciudad. El diseño fue abordado en tres etapas: a) Exploración del contexto y documentación, para verificar la relevancia del tema en la comunidad; b). Selección del modelo didáctico y c). Construcción del módulo. Finalmente, se presenta a modo de ejemplo una actividad de juego de roles, diseñada bajo el enfoque de enseñanza-aprendizaje de educación para la sostenibilidad y cuestiones socio-científicas (CSC) que permite reflexionar cada uno de los problemas ambientales tratados.

Palabras clave: Módulo didáctico, problemas ambientales, educación para la sostenibilidad.

Abstract: The need to design materials that promote reflection about environmental problems, makes educational institutions assume promoter scenarios in the creation of educational strategies to address this issue, so this paper presents the design process of a training module called "Reflect on Environmental Problems of the Municipality of Tunja", which aims to recognize the most important environmental problems affecting this

$1 \quad$ Licenciado en Ciencias Naturales y Educación Ambiental de la Universidad Pedagógica y Tecnológica de Colombia. Actualmente labora en la Corporación Autónoma Regional de Boyacá CORPOBOYACÁ desempeñando labores como técnico operativo en el sector de Residuos Peligrosos. Autor del modulo de Reflexionemos sobre los Problemas Ambientales del Municipio de Tunja que se encuentra incluido en la cartilla Tunja ciudad de riqueza natural: conozcamos y valoremos nuestros recursos.

2 Magister en Docencia de la Química Universidad Pedagógica Nacional. Actualmente labora como docente facultad de Educación de la Universidad Pedagógica y Tecnológica de Colombia, anteriormente laboraba como docente de Química y Bioquímica de la Universidad de Boyacá. Ha participado como ponente en congresos nacionales e internaciones sobre Pedagogía, Educación, Didáctica de las ciencias. Ha escrito relacionados con el Habilidades de pensamiento crítico, cuestiones socio científicas y Ambientes de aprendizaje en Revistas como Luna Azul de la Universidad de Caldas y la Revista Qurriculum en España. 
city. The design was divided into three stages: $a$. Context and documentation research, in order to verify the relevance of the topic in society. b. Approach Selection. c. Module Construction. Finally, is presented by way of example, a role-playing activity developed with the focus on teaching and learning in education for sustainability and socio-scientific issues (CSC) that allows to reflect each of the treated environmental problems.

Keywords: Training module, environmental issues, education for sustainability.

\section{Introducción}

Los impactos y problemas ambientales no son exclusivos de la época actual, en tiempos del imperio Romano se presentaban problemas como la erosión. Hacia el año 1306 en Inglaterra ya existían algunas prohibiciones sobre la quema de carbón por la contaminación atmosférica que se producía (Bordehore, 2001). En épocas más próximas como la revolución industrial se describe problemáticas globales que han tenido un incremento exponencial afectando los recursos naturales existentes en el planeta.

Torres (2002) indica que los problemas ambientales hacen evidente las desarmonías, tanto en el sistema natural, como en el sistema sociocultural y sus impactos tienen consecuencias globales como el calentamiento global, perdida de la biodiversidad, pobreza, contaminación, entre otras. Ulloa (2007) señala que los problemas ambientales permiten hacer lecturas sobre la calidad de las relaciones de los grupos humanos, con los sistemas naturales a través de los cuales se desarrollan sus dinámicas sociales y culturales. Por ello, toma relevancia el estudio de los problemas locales que pueden repercutir en los problemas globales.

Desde esta mirada en el ámbito educativo, específicamente en espacios de formación docente, surge la pregunta ¿Cómo abordar en el aula los problemas ambientales locales, que superen una visión lineal y mecanicista, y más bien permitan un proceso de reflexión crítica que lleve a formar actitudes sobre la realidad? Al respecto, Batalla (1997); Meinardi, Adúriz y Revel, (2002) hacen énfasis en la necesidad de promover estrategias didácticas en los entornos urbanos y rurales, para tomar en cuenta la relación reciproca que se da entre el patrimonio natural y el patrimonio edificado, lo cual enriquece una imagen urbana que permite formar una cultura preocupada por el equilibrio ambiental.

Así mismo, Solís (2006); Wrigth (2010) indican, que la educación como proceso y la escuela como institución, juegan un papel esencial en la búsqueda de alternativas sostenibles al ambiente, que proporcionan el conocimiento, las habilidades y motivaciones necesarias para dar al individuo herramientas que le permitan interpretar el mundo y una actuación social consecuente con sus necesidades y exigencias.

Cuello (2003) manifiesta que los problemas ambientales son un recurso para el aprendizaje en el aula, señala algunas implicaciones didácticas, p.e utilizar situaciones auténticas, articular el entorno con intereses de los estudiantes. Esto motiva a movilizar ideas procedentes del ámbito ideológico, cotidiano y científico.

Lo anterior, lleva a pensar como diseñar estrategias didácticas que permitan el conocimiento de las afectaciones ambientales en una localidad. Por ello, este trabajo presenta el diseño en la elaboración de un módulo didáctico denominado: "Reflexionemos sobre los Problemas Ambientales del Municipio de Tunja”, que tiene como finalidad el reconocimiento de los problemas ambientales más relevantes que afectan a esta ciudad, siendo una oportunidad para reflexionar y ejercer cambios de conductas de las actuaciones humanas en el ambiente. 


\section{Metodología}

En esta experiencia, se elaboró un módulo didáctico relacionado con los principales problemas ambientales del municipio de Tunja, una ciudad colombiana, denominado: Reflexionemos sobre los Problemas Ambientales del Municipio de Tunja.

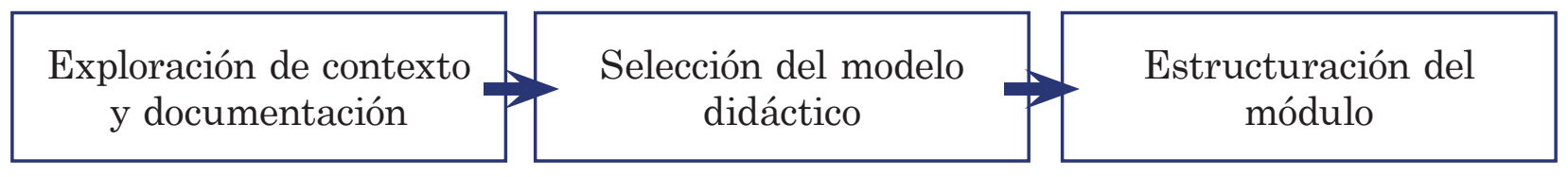

Figura 1. Etapas de diseño del módulo.

Nota: Realizado por los autores.

Para el diseño del módulo (Figura 1) se planearon las siguientes etapas:

En primer lugar, se realizó una exploración de contexto y documentación. Se efectuó una indagación inicial con aspectos generales que permitió identificar los principales problemas ambientales del municipio de Tunja. Se seleccionó un grupo de 20 personas de la comunidad tunjana, considerando los referentes metodológicos de Krueger y Casey (2000) y Korman (1986) y que dan uso a este tipo de técnicas para la búsqueda de información.

La elección de los participantes se hizo de acuerdo a criterios como:

- Que habiten en el municipio de Tunja en un tiempo mayor a seis (6) años.

- Personas mayores de 14 años.

- Con diferentes ocupaciones que comprenden desde estudiantes (de universidad y secundaria), constructores, amas de casa, empleadas de servicio, panaderos, pasteleros, transportadores y trabajadores independientes.

La búsqueda de información se realizó en documentos de la Alcaldía Mayor de Tunja, páginas de revistas especializadas, artículos científicos, trabajos de tesis de la Universidad Pedagógica y Tecnológica de Colombia (UPTC), legislación ambiental y noticias ambientales por medios de comunicación locales y nacionales.

En la segunda etapa se realizó la selección del modelo didáctico Educación para la sostenibilidad, como enfoque de enseñanza-aprendizaje para potenciar en las personas de todas las edades la responsabilidad de crear y disfrutar un futuro sostenible (Cambers, Chapman, Diamond, Down, Griffith, y Wiltshire, 2008).

En la tercera etapa se estructuró los contenidos del módulo y se presenta información referente a cada uno de los principales problemas ambientales.

Finalmente, como estrategia para la consolidación conceptual y actitudinal, se planeó una actividad de juego de roles, para abordar los problemas ambientales en la ciudad, desde el 
enfoque de cuestiones socio-científicas (CSC) para motivar un cambio actitudinal y considerar otros temas desde la enseñanza de las Ciencias Naturales (Torres, 2011).

\section{Resultados y discusión}

Los resultados se presentan considerando los momentos metodológicos señalados.

\section{Diagnóstico}

El diagnostico permitió identificar los problemas ambientales en el municipio de Tunja. En las apreciaciones de los participantes se vinculan consecuencias inmediatas de acciones humanas como basuras, deforestación y calentamiento global a los problemas ambientales. Un ejemplo de estas respuestas son las siguientes:

P3: "Las basuras, la contaminación del aire, falta de arborización, calentamiento global". P6: "Es un daño que le ocurre al ambiente por efecto de materiales usados por el hombre para su beneficio"; P10: "Cualquier cosa que afecte la naturaleza y el equilibrio del ambiente"; "P15: "Son situaciones que generan un impacto negativo en el medio ambiente".

Un ejemplo de respuesta, que permite identificar otros elementos en relación a los problemas ambientales la indica el participante 9: P9: "Es una situación en particular que afecta la humanidad y al planeta en los tres componentes del ambiente: la naturaleza, la sociedad y la cultura". Al respecto, Torres (2002) indica que un problema ambiental hace evidente la desarmonía, en el sistema natural y sociocultural. Lo anterior, denota la importancia de la inclusión de aspectos sociales para superar intereses dominantes e individuales que impiden pensar las implicaciones ambientales globales que tienen.

En la pregunta De los problemas ambientales mencionados ¿Cuál cree usted que es el más preocupante? Esta permitió definir los principales problemas ambientales que fueron presentados en el contenido del módulo, como se ilustra en la tabla 1.

\section{Tabla 1.}

Identificación de los principales problemas ambientales

\begin{tabular}{cc}
\hline PROBLEMA AMBIENTAL & JUSTIFICACIÓN \\
\hline Contaminación auditiva & $\begin{array}{c}\text { P8: "Debido a que cada vez aumenta más y genera enfermedades en el } \\
\text { sistema auditivo y se incrementa por los trancones y a la baja capacidad de } \\
\text { las vías." }\end{array}$ \\
Erosión & $\begin{array}{c}\text { P5: "Es un factor que afecta al ambiente y ha sido ignorado sin tener en } \\
\text { cuenta que perjudica a uno de los recursos más importante como es el suelo" }\end{array}$ \\
$\begin{array}{c}\text { Contaminación por } \\
\text { escombros }\end{array}$ & $\begin{array}{c}\text { P1: "Estos dan un muy mal aspecto y permanecen mucho tiempo } \\
\text { en un solo sitio, ocasionando que el terreno se comprima e impida que se } \\
\text { desarrolle las plantas". }\end{array}$
\end{tabular}


Residuos sólidos

Contaminación atmosférica

El manejo de los plaguicidas
P10:"Se producen constantemente y algunas personas con poca conciencia ambiental no lo depositan donde deben y van a parar a los ríos, también pueden ocasionar taponamiento de las alcantarillas".

P13"Contaminan la naturaleza pues hay desechos de todas clases, muchos sitios son tomados como botaderos, no hay una buena conciencia ambiental de la importancia del reciclaje".

P18:"No hay control de las autoridades, ni educación por parte de los dueños de los carros que ignoran las revisiones que se deben realizar para disminuir los gases contaminantes, pues estos generan calentamiento global y destrucción de la capa de ozono."

P17: " El humo afecta a las personas que salen a caminar pues lo aspiran y perjudican su salud"

P14: "Cuando se realiza un mal manejo de estos químicos generan un detrimento de las fuentes hídricas, suelo, fauna, flora e inclusive puede generar enfermedades."

Así mismo, los participantes indican que una de las formas que se deberían implementar para disminuir los problemas ambientales en el municipio de Tunja, son las capacitaciones y la creación de programas por parte del gobierno municipal que traten las cuestiones ambientales, para generar una conciencia en el cuidado de la naturaleza y hacer frente a los problemas ambientales en este municipio. De la misma forma, los participantes resaltan la importancia que tienen los colegios en los procesos de educación ambiental.

Estas consideraciones permiten estar de acuerdo a lo señalado por Rivarosa y Perales (2006) sobre la importancia de hacerse parte del problema y no pensar que las soluciones esta en los otros. El autor describe a los otros como los que gobiernan y toman decisiones políticas. Esta referencia hace énfasis en la necesidad que cada ciudadano reconozca su ambiente con sus múltiples dinámicas. Por tanto, se requiere originar un cambio de comportamiento y de responsabilidad ambiental en cada persona.

Desde esta perspectiva se eligió el modelo de Educación para la sostenibilidad porque ayuda a tratar los principales problemas ambientales del municipio de Tunja. Rescata la importancia de conocerlos en todos los niveles escolares y en todos los sectores de la comunidad. Favorece en las personas cambios de actitudes, que contribuyen en la adopción de medidas para la sostenibilidad en las actividades que a diario se realizan. Enfatiza en la necesidad que las personas de cualquier entorno reconozcan su ambiente próximo con sus afectaciones para promover actitudes criticas frente a los problemas ambientales.

\section{La estructuración del módulo}

El módulo se propone como una herramienta didáctica para contribuir a una educación ambiental a todas las personas. El contenido del módulo se plantea con los siguientes cuestionamientos: ¿Qué es un problema ambiental? ¿Por qué nacen los problemas ambientales? ¿Cuáles son los principales problemas ambientales de la ciudad de Tunja? Y por último, se presenta la estrategia de consolidación de conceptos. 
A continuación se presentan algunos de los ítems:

\section{Título}

El título propuesto para el modulo se denominó "Reflexionemos sobre los Problemas Ambientales del Municipio de Tunja" (Figura 1), surge como iniciativa de construcción de actitudes favorables para fortalecer la interacción hombre-sociedad-naturaleza y desarrollar competencias básicas que permitan conocer y solucionar los problemas ambientales (Ministerio de Educación Nacional, 1998).

$\mathrm{El}$ fin de exponer los problemas ambientales se hace para que la comunidad y en especial las instituciones educativas juzguen que se requiere una escuela que permita la participación activa del niño en primera instancia, y de toda la comunidad en la construcción del conocimiento para encontrar alternativas de solución acordes con su problemática ambiental (Torres, 1996).

\section{REFLEXIONEMOS SOBRE LOS PROBLEMAS AMBIENTALES DEL MUNICIPIO DE TUNJA}

Las problemáticas ambientales han estado presentes desde tiempos antiguos en la vida de los habitantes de la tierra, afectando los sistemas que conforman el ambiente (el cultural, el social y el natural). Desde esta perspectiva conocer y reflexionar los principales problemas ambientales del municipio de Tunja permitirá generar acciones responsables en los ciudadanos, además de contribuir a la comprensión de las relaciones de interdependencia de los seres humanos con su entorno; es así como se hace necesaria la generación de actitudes de valoración y respeto por los recursos naturales de nuestra ciudad y a partir de este modulo queremos ilustrar los principales problemas ambientales que afectan el municipio de Tunja y a la vez presentar las alternativas de solución que han generado los estamentos gubernamentales de la ciudad.

Figura 2. Presentación del módulo de problemas ambientales.

Nota. Becerra y Torres, 2011.

Posterior a la presentación del módulo se presenta una conceptualización respecto a la denominación de un problema ambiental:

\section{¿Queé es un problema ambiental?}

Se abordó la conceptualización de un problema ambiental (Figura 2). La definición mostrada enmarca gran cantidad de factores que influyen en las causas, consecuencias y su evolución a través de la historia; entendiendo que el problema ambiental es el resultado de los impactos producidos por transformaciones o modificaciones de la actividad humana, para la satisfacción de necesidades que tiene implicaciones en el deterioro de los componentes del ambiente (Torres, 2002).

A través de este concepto se pretende observar la verdadera dinámica de los problemas ambientales para que sean entendidos dentro de la compleja trama de las interrelaciones 
entre el hombre como ser social y la naturaleza (Marín, 2009). De esta manera, el contenido es aprovechado por los docentes como un instrumento que facilite el conocimiento de los problemas ambientales de un modo más amplio. Rivarosa y Perales (2006) indica que si los problemas ambientales son introducidos dentro del currículo, se constituyen en herramientas para que se entiendan que son significativos y funcionales en la vida de las personas. Si bien, el estudio de estos depende de muchas variantes, le deben al estudiante tomar sentido y verlos como asuntos reales, es decir aplicables a la vida para movilizar contenidos culturales socialmente relevantes.

\section{CQUÉ ES UN PROBLEMA AMBIENTAL?}

Un problema ambiental es aquel producido por la humanidad a lo largo de la historia; el cual afecta al planeta y a todos sus habitantes sin importar color de piel, creencia religiosa, nacionalidad, estrato social etc. Este a su vez no es fácil de perobir, dado que es consecuencia de acciones continuas de las personas en el ámbito: económico, social, cultural, político y natural.

Considerando lo anterior, presentamos la siguiente clasificación de los problemas ambientales de acuerdo a Ortega Et al (2007):

Figura 3. Conceptualización de un problema ambiental.

Nota. Becerra y Torres, 2011.

\section{¿Por qué se originan los problemas ambientales?}

Entender el origen de los problemas ambientales para un estudiante no es fácil. Frente a esto Rivarosa y Perales (2006) indican que la mala planificación curricular también ha ocasionado que el estudiante no sea consciente de las causas que los producen. Se cree que la solución de estos, se encuentra en los que gobiernan y los que toman decisiones políticas y económicas, cuando también es importante pensar sobre las acciones individuales con responsabilidad social. De igual forma, es necesario reflexionar sobre la multidisciplinariedad y la interculturalidad, intentando siempre construir marcos de referencia integrales, producto de las dinámicas entre los saberes.

Acercar al estudiante al nacimiento de los problemas ambientales lo vincula aún más con su entorno próximo. Le proporciona herramientas que les permite construir una ética ambiental para comprender que la naturaleza aunque limitada, es de un valor inestimable, lo cual implica que el hombre debe pensar y repensar la calidad de sus relaciones con el medio. (Ministerio de Educación Nacional, 1998). Figura 4. 


\section{¿PORQUÉ NACEN LOS PROBLEMAS AMBIENTALES?}

Los 6500 millones de habitantes del mundo, los 46 millones personas en Colombia y los 164.676 individuos de Tunja somos los responsables de mantener este planeta libre de contaminación.
La mayoría de los problemas ambientales han surgido debido a los excesos de consumo de las personas y a los grandes niveles de contaminación provocados por los modelos industriales. Estudios de Vilches (2003), reflejan que no se ha tenido una buena relación y responsabilidad con la naturaleza, ya que esta ha sido vista como fuente inagotable de recursos, sin considerar las consecuencias que dejan acciones diarias como: la utilización del agua, el consumo de productos; de la misma manera se deben educar en una cultura etica con el contexto. etc.

Figura 4. Contextualización sobre problemas ambientales

Nota. Becerra y Torres, 2011.

Conforme al desarrollo del módulo se ilustran los principales problemas ambientales que se presentan en el municipio de Tunja (Figura 5 y 6.) exponiendo los más trascendentales, según lo expresado por el grupo de habitantes participantes en la fase diagnostica. En esta medida, se hace énfasis en la participación activa de toda la comunidad para encontrar alternativas de solución acordes con su problemática ambiental en particular.

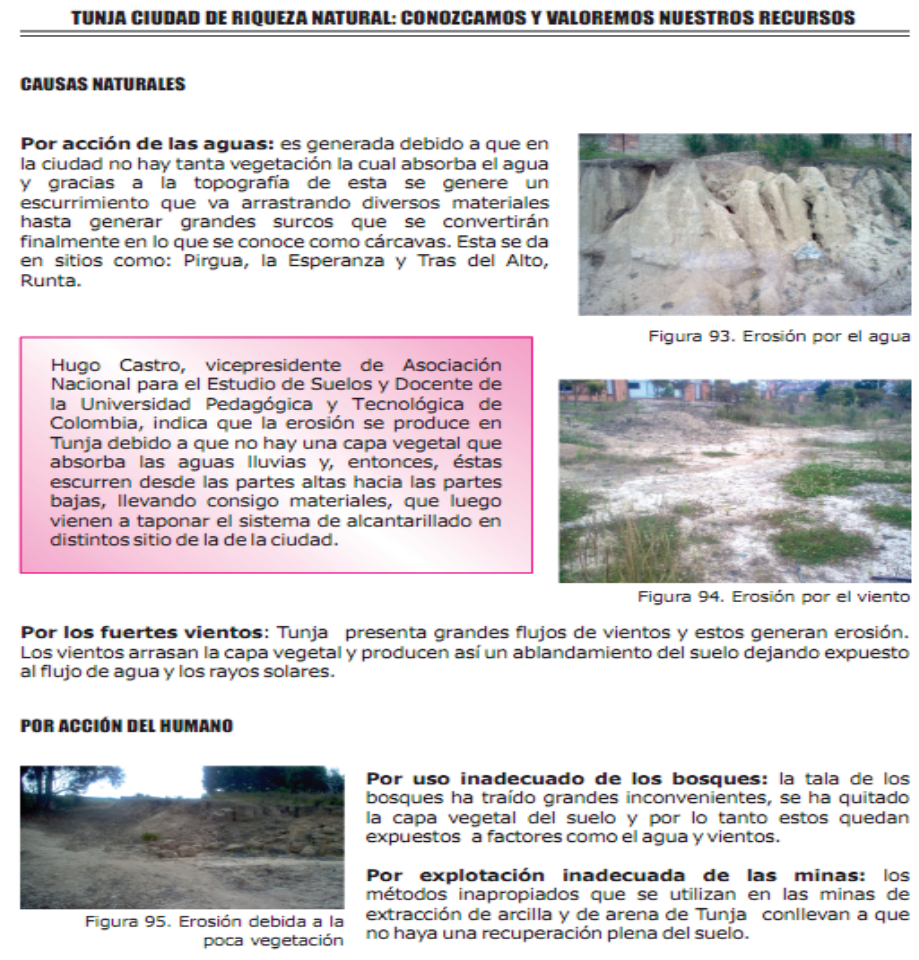

Figura 5. Ejemplo del abordaje de uno de los problemas ambientales (la erosión) Nota. Becerra y Torres, 2011. 
TUNJA CIUDAD DE RIQUEZA NATURAL: CONOZCAMOS Y YALOREMOS NUESTROS RECURSOS

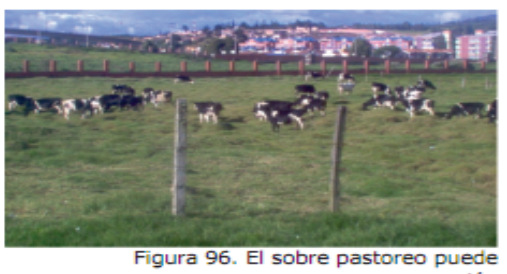

generar erosión

Por la sobreutilización agrícola y el pastoreo: en el caso del municipio de Tunja es muy frecuente. Al realizar el arado la tierra queda expuesta los rayos solares, el viento y el agua.

Como podemos evidenciar son muchas más las causas erosivas por acción de los humanos que por causas naturales, por lo cual se requiere que empecemos a formar una conciencia ambiental que nos permita estar en armonía con la naturaleza.
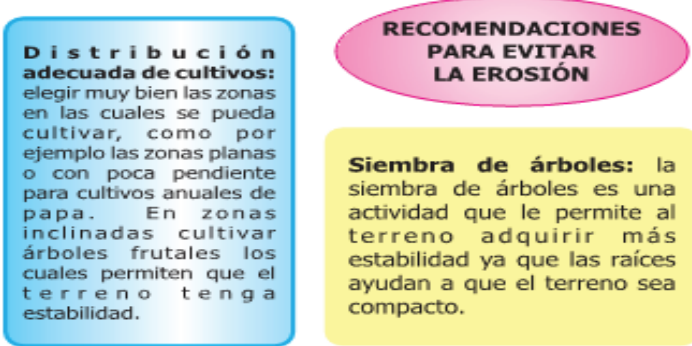

Siembra de árboles: la siembra de árboles es una actividad que le permite al terreno adquirir más terreno adquirir más ayudan a que el terreno sea compacto.

Distribución de zonas de pastoreo: tener parcelado un terreno el cual es destinado para el destinado para el pastoreo permite el cambio de sectores a los animales, con el fin de realizar una rotación para que el terreno tenga la oportunidad de recuperarse.

\section{ESCOMBROS}

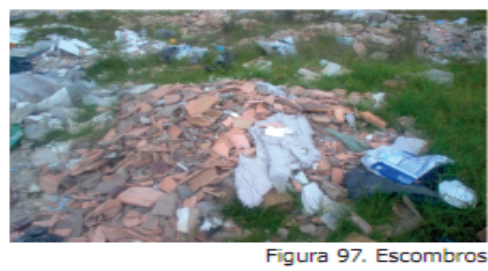

Los escombros son conocidos comúnmente como aquellos residuos o sobrantes que quedan después de haber realizado algún tipo de construcción.

En Tunja el problema de los escombros es muy marcado debido a la expansión urbana, de todas las construcciones quedan sobrantes de materiales que son depositados en lugares no adecuados como: lotes abandonados, carreteras, parques y sectores aledaños a la obra.

Figura 6. Abordaje de uno de los problemas ambientales (la erosión) Nota. Becerra y Torres, 2011.

A continuación, en la tabla 2 , se presentan los problemas ambientales abordados en el módulo didáctico. 


\section{Tabla 2}

\section{Análisis de los problemas ambientales abordados en el módulo didáctico}

\begin{tabular}{cl}
$\begin{array}{c}\text { Problema } \\
\text { ambiental }\end{array}$ & \multicolumn{1}{c}{ Contextualización } \\
\hline $\begin{array}{c}\text { Contaminación } \\
\text { Auditiva }\end{array}$ & $\begin{array}{l}\text { El ruido siempre ha sido un problema ambiental } \\
\text { importante para el ser humano (Berglund et } \\
\text { al., 1999). Este tiene su causa principal en las } \\
\text { actividades humanas y sus fuentes son: el tránsito } \\
\text { automotor, ferroviario y aéreo, la construcción y } \\
\text { obras públicas. }\end{array}$ \\
\end{tabular}

Garrini y Leonardini (2006) indica que el ruido es un problema esencialmente del sector urbano debido a que los componentes naturales han sido cambiados por lo que se conocen como selvas de concreto, que son generadoras de sonidos incómodos.

Erosión La erosión en Tunja, se presenta tanto por causas naturales como antrópicas, siendo las primeras menos frecuentes pues hacen parte de ciclos nórmales de la naturaleza como la escorrentía y el viento (Sotelo, 2003).

Las causas antrópicas se presentan por diferentes factores que contribuyen al deterioro del suelo. A nivel mundial este problema está acompañado de la pérdida de grandes extensiones de bosques, esto influenciado principalmente por el desarrollo agrícola (Secretaria de Educación Pública, 1999). Sin olvidar, que este sector de la economía, también es afectado por la disminución de la capacidad del suelo para permitir el crecimiento adecuado de los cultivos (López- Bermúdez, 1990).

Los La generación de escombros actualmente parece inevitable Escombros pues constantemente a nivel mundial se derrumban edificaciones para dar paso a otras más modernas.

Tunja no es ajena a este aspecto atraviesa un gran desarrollo urbanístico y vial ocasionando un gran incremento de materiales sobrantes de construcciones en gran parte de la ciudad. Si este desarrollo se efectúa de manera desordenada, es contemplado dentro de los factores que ocasionan una crisis planetaria, pues contribuye aún agotamiento de los recursos (Girardet, 2001).
La identificación de este problema permite abordar una visión amplia de los efectos del ruido en la salud como fatiga auditiva, disminución de la agudeza auditiva, alteraciones del ritmo cardíaco, fluctuaciones en la presión arterial, alteraciones en la amplitud respiratoria, efectos sobre el sistema nervioso, perturbaciones en el sueño, disfunciones en el aparato digestivo.

Por tanto, se pueden plantear actividades que permitan identificar los sitios de mayor contaminación auditiva en el barrio, colegio etc.

Mostrar este problema permite reflexionar acerca del recurso suelo. Lugar donde se llevan a cabo procesos dinámicos de múltiples organismos.

Se puede discutir sus implicaciones en la infraestructura del crecimiento de una ciudad. También generar discusiones que permitan hacer comparaciones de las disminuciones de hectáreas de bosques en la región y sus consecuencias.

Discutir las implicaciones del crecimiento de la agricultura, lo cual permite articularlo con otros problemas como el uso de plaguicidas.

Este tema permite abordar aspectos referidos a la legislación para la disposición adecuada de los escombros.

Serrano y Ferreira (2009) indican que en Colombia, aunque existe normatividad que regula la disposición de escombros, la gestión integral de estos ha sido muy deficiente. Esto permite posibilitar espacios de investigación que planteen el desarrollo de tecnologías, para el aprovechamiento de los escombros como agregados de concreto para el desarrollo de adoquines, mampuestos, concreto para andenes y sardineles.

Nota: Realizado por las personas autoras 
Una vez abordado los distintos problemas ambientales en el ámbito local se propone una estrategia de consolidación de conceptos.

\section{Estrategia de consolidación de conceptos.}

Como estrategia para la consolidación de conceptos se planteó la actividad titulada "Aprendamos a Controlar los Problemas Ambientales de Nuestro Municipio" (Anexo 1) desde un juego de roles, donde los participantes toman posturas críticas desde un rol, y facilita comprender la conceptualización del problema ambiental y la importancia de participar de manera democrática en la solución de estos.

La reproducción de ciertas situaciones reales por medio del uso de simulaciones obtenidas permiten abordar otros aspectos como la ciencia, la tecnología y la cultura, además de promover reflexiones en los estudiantes frente a asuntos sociales, éticos y ambientales que pueden originar acciones encaminadas hacia la sostenibilidad y cuya intención es poner de manifiesto las interacciones entre los distintos agentes sociales (Sánchez, 2009).

A través del juego de roles se pretende mostrar que los problemas ambientales no pueden ser tomados como simples dificultades con una solución. Estos están lejos de ser simples inconvenientes a los que se les puede dar solución sin esfuerzo alguno (Rivarosa y Perales, 2006). A partir del uso de simulaciones acompañado de las CSC se fortalece vínculos entre la sociedad y la ciencia para entender que se deben buscar soluciones colectivas a los problemas socio-ambientales.

En este sentido, las CSC hacen posible la transformación completa de un tema tradicional ya existente, en una propuesta que requiere pensamiento crítico y toma de decisiones; posibilitando reflexiones profundas sobre el compromiso que deben adquirir los docentes y los futuros docentes, respecto a la contribución de una educación con acciones de ciudadanía (Torres, 2011). De otra parte, Gil y Vilches (2006) indican la relevancia que toma la enseñanza de los problemas ambientales en la escuela, que va más allá del aprendizaje de conceptos y busca garantizar una educación integral.

\section{Impactos de material en docentes en ejercicio}

Se consideró que una de las fases importantes en el diseño de cualquier material educativo, tiene que ver con generar grupos de discusión entre los mismos docentes. Por ello, en esta fase se divulgo el material diseñado a 12 docentes de una institución educativa. Se efectuó una presentación en Power Point sobre los problemas ambientales y se mostró el material elaborado. Se aplicó un cuestionario de 5 preguntas para recoger apreciaciones de los docentes frente al material.

En síntesis de esta primera socialización, los docentes indicaron que se constituye en un material para incentivar a los estudiantes a un mayor compromiso y responsabilidad a nivel socio-cultural y natural. Las actividades propuestas permiten reflexionar sobre la importancia, reconocimiento, conservación y uso sostenible de los recursos naturales para 
mitigar los problemas ambientales que hoy se presentan en el municipio. Señalan que el material es una guía para el docente.

Algunas afirmaciones fueron Pr3:"El material trae los temas y la documentación y preparación para la dinámica del trabajo con los estudiantes"; Pr11: "Lo utilizaría como material de consulta y reflexión"; Pr7: "El modulo es adecuada para tratar los tema ecológicos de la ciudad y el municipio" Pr5: "Este tipo de recursos o herramientas didácticas son muy importantes, puesto que generan una ayuda al docente para reconocer los principales problemas ambientales del municipio de Tunja y para el estudiante porque explora sobre la existencia de diferentes recursos y del cómo pueden actuar frente a posibles soluciones de los perjuicios ambientales"

Así mismo, los docentes hacen énfasis en el abordaje de los problemas ambientales como tema transversal al currículo, ejemplo de esto lo indica el profesor Pr13:" Es necesario trabajarlo en las diferentes áreas y no solo en biología"; Pr2: "Más que los contenidos escritos es importante concientizar a los niños, jóvenes, adultos, de la importancia de enriquecer nuestro entorno y cuidarlo para llenar al municipio de plantas que atraen más vida animal".

Esta valoración permitió conocer algunos aspectos del módulo por parte de un grupo de docentes, que consideró el material como herramienta de apoyo para abordar los problemas ambientales, dentro de la enseñanza de los diferentes contenidos curriculares.

\section{Consideraciones finales e implicaciones didácticas}

Desde la experiencia descrita se pretende mostrar el papel de docente como diseñador y productor de material educativo, punto de partida para el conocimiento de los problemas ambientales. Sin embargo, se sugiere que esta función no se reduzca a aspectos conceptuales, sino también dar lugar a espacios procedimentales y actitudinales en las instituciones educativas. Por tanto, debe pensarse que el diseño de material didáctico, involucre a padres y comunidad en general, esto para no limitar la capacidad de acción de los adultos que pueden mejorar conductas ambientales desde los hogares. De igual forma, se resalta la necesidad de generar grupos de discusión entre docentes para discutir el impacto y diseño de cada material didáctico.

El material didáctico diseñado por los docentes acerca de los problemas ambientales toma gran relevancia, porque puede contribuir a que la sociedad que en ocasiones ha ignorado los llamados de distintas organizaciones, se haga participe de la solución de los problemas ambientales y proporcionen situaciones donde se reconozcan y reflexionen sus implicaciones. Además, es un mecanismo para generar actitudes ambientales que superan una visión limitada y conecten al individuo con su entorno ambiental próximo que contribuye a una visión sistémica del ambiente.

Desde lo propuesto anteriormente se considera fundamental abordar problemas inmediatos. En este caso, se presentó como ejemplo el tipo de problemas ambientales de una ciudad colombiana, lo cual aporta al conocimiento de problemas específicos de un contexto próximo para encaminar hacia actitudes ambientales en relación al entorno. Esto evitaría cuestiones generales que promueven solo el enfoque informativo pero no actitudinal. Lo anterior sería una contribución a transversalizar la educación ambiental desde un plano de la realidad actual que permite no dejar la información solo en el aula sino llevarla a la comunidad en general. 
Se sugiere utilizar el enfoque de las CSC como herramienta que facilite conocer y abordar los problemas ambientales de un modo más amplio. No limitarse simplemente a un contenido ya establecido en los libros de texto, sino buscar relacionarlos con actividades en el ámbito natural, social, económico, político entre otros. Esta herramienta pedagógica y didáctica puede ser una contribución al conocimiento de los problemas ambientales y promover actitudes éticas y de ciudadanía.

De la misma manera indicamos que el juego de roles contribuye a tomar posturas de actores inmediatos en el contexto y sentirse participes de un problema ambiental. Estos permiten ingresar a un ambiente participativo con argumentos desde sus propios puntos de vista. El juego de roles facilita el trabajo en equipo que permite valorar las implicaciones desde diferentes ocupaciones en la sociedad.

La experiencia a modo de ejemplo permitió indagar acerca de la existencia de los hornos de fabricación de ladrillos que tienen implicaciones en problemas de contaminación atmosférica, sumado a la contaminación generada por cada uno de los vehículos que transitan a diario en el municipio. Así mismo, se sugiere realizar diferentes tipos de estrategias de educación ambiental sobre el uso y afectación de los plaguicidas. Programas educativos que permitan abordar las implicaciones de los plaguicidas en fuentes hídricas, especies silvestres, suelo y aire.

\section{Referencias}

Bonfill Batalla, G. (1997). Nuestro patrimonio cultural: un laberinto de significados. En El patrimonio nacional de México. (pp. 28-56) México: CONACULTA y FCE.

Becerra, J., y Torres, N. (2011). Reflexionemos sobre los problemas ambientales del municipio de Tunja. En Alcaldía Mayor de Tunja: Secretaria de Desarrollo (Ed.), Tunja ciudad de riqueza natural: Conozcamos y valoremos nuestros recursos (pp. 79-100).Tunja, Colombia: Alcaldía Mayor de Tunja.

Berglund, B., Lindvall, T., y Schwela, D. (Eds.). (1999). Guías para el ruido urbano. Londres: Organización Mundial de la Salud. Recuperado de-http://www.bvsde.paho.org/bvsci/e/ fulltext/ruido/ruido2.pdf

Bordehore, C. (2001). Problemas ambientales, problemas humanos. En A. Aledo, J. Domínguez (Dirs.), Sociología Ambiental (pp. 321-355). Granada, España: Grupo Editorial Universitario.

Cambers, G., Chapman, G., Diamond, P., Down, L., Griffith, A., y Wiltshire, W. (2008). Educación para el desarrollo sostenible. Aportes didácticos para docentes del Caribe. Santiago, Chile: OREALC/UNESCO.

Cuello, A. (2003). Problemas ambientales y Educación Ambiental en la escuela. Reflexiones sobre Educación Ambiental II. Artículos publicados en la Carpeta Informativa del 
CENEAM 2000-2006, 91-113.Recuperado de http://www.magrama.gob.es/es/ceneam/ recursos/documentos/reflexiones-educacion-ambiental-carpeta-ceneam tcm7-13563.pdf

Garrini, D., y Leonardini, R. (2006). Contaminación acústica. Como agente generador de disfonía profesional en la actividad docente. Santa Fe, Argentina: Grupo Sancor Seguros.

Gil, D., y Vilches, A. (2006). Algunos obstáculos e incomprensiones en torno a la sostenibilidad. Revista Eureka Sobre la Enseñanza y Divulgación de la Ciencias, 3(3), 507-516. Recuperado de http://www.uv.es/vilches/documentos\%20enlazados/Eureka\%202006.pdf

Girardet, H. (2001). Creando ciudades sostenibles. Valencia, España: Tilde.

Korman, H. (1986). The Focus Group Sensign. Dept. Of Sociology. [La detección de grupos focales. Departamento de sociología] New York: SUNY at Stony Brook.

Krueger, R., y Casey, M. (2000). Focus groups: A practical guide for applied research (3 ${ }^{\mathrm{a}}$ ed). [Los grupos focales: una guía práctica para la investigación aplicada ( $\left.\left.3^{\mathrm{a}} \mathrm{ed}\right)\right]$ California: Thousand Oaks.

López-Bermúdez, F. (1990). Soil erosion by water on the desertification of a semi-arid Mediterranean fluvial basin: the Segura basin, Spain. Agriculture, Ecosystems \& Environment, 33(2), 129-145. DOI: http://dx.doi.org/10.1016/0167-8809(90)90238-9

Marín, L. (2009). La gestión de problemas ambientales y el trabajo social comunitario a nivel local. Su incidencia en la capacitación de actores locales en la comunidad "La Cabaña" del municipio del Pinar del Río. (Tesis de Maestría, Universidad Pinar del Río, Pinar del Río, Cuba). Recuperado de http://flacsoandes.org/dspace/bitstream/10469/1636/1/La\%20 gesti\%C3\%B3n\%20de \%20problemas\%20ambientales\%20y\%20el\%20trabajo...\%20 Lisbert\%20Roig.pdf

Meinardi, E., Adúriz, A., y Revel, A. (2002). La Educación Ambiental en el aula. Una propuesta para integrar contenidos multidisciplinares a través de la argumentación. Investigación en la Escuela, (46), 93-103.

Ministerio de Educación Nacional. (1998). Ciencias naturales y educación ambiental: lineamientos curriculares. Referentes teóricos, implicaciones pedagógicas y didácticas aplicaciones. Bogotá: Ministerio de Educación Nacional.

Rivarosa, A., y Perales, J. (2006). La resolución de problemas ambientales en la escuela y en la formación inicial de maestros. Revista Iberoamericana de Educación, (40), 111-124. Recuperado de http://www.ambiente.gov.ar/infotecaea/descargas/rivarosa03.pdf 
Sánchez, M. (2009). El juego y otras actividades lúdicas para la educación ambiental en los escolares. Revista Digital Innovación y Experiencias Educativas, (14), 1-16. Recuperado de: http://www. csiif.es/andalucia/modules/mod ense/revista/pdf/Numero 14/MARIA\%20ANGELES SANCHEZ 1.pdf

Secretaria de Educación Pública. (1999). La educación ambiental en la escuela secundaria: Guía de estudio. Mexico D.F: Secretaria de Educación Pública.

Serrano, M., y Ferreira, S. (2009, septiembre). Aprovechamiento de los escombros para la producción de concreto. II Simposio Iberoamericano de Ingeniería de Residuos. Barranquilla, Colombia: Uninorte.

Solís, E. T. (2006). Algunos elementos del proceso de construcción de la educación ambiental en América Latina. Revista Iberoamericana de educación, (41), 69-81.

Sotelo, A. (2003, 24 marzo). Planes ambientales para atacar la erosión en Tunja. El Tiempo, Seccion Archivo. Recuperado de http:/www.eltiempo.com/archivo/documento/MAM975926

Torres, M. (1996). Educación Ambiental. La dimencion ambiental: Un reto para la educación de la nueva sociedad.Proyectos Ambientales escolares. Bogotá: Ministerio de Educación Nacional.

Torres, M. (2002). Reflexión y acción: el diálogo fundamental para la educación ambiental. Teoría y práctica. Bogotá: Ministerio de Educación Nacional y Ministerio del Medio Ambiente.

Torres, N. (2011). Las cuestiones socio-científicas: una alternativa de educación para la Sostenibilidad. Revista Científica Luna Azul, (32), 80-85. Recuperado de http://www. scielo.org.co/pdf/luaz/n32/n32a04.pdf

Wrigth, T. (2010). University president's conceptualizations of Sustainability in Higher Education. [Conceptualizaciones del presidente de la Universidad de la Sostenibilidad en la Educación Superior] International Journal of Sustainability in Higher Education 11 (1), 61-73. DOI: http://dx.doi.org/10.1108/14676371011010057

Ulloa, A. (2007). La articulación de los pueblos indígenas en Colombia con los discursos ambientales, locales, nacionales y globales. En Formaciones de indianidad. Articulaciones raciales, mestizaje y nación en América Latina (279-326). Recuperado de http://www. ram-wan.net/restrepo/inv-antrop/ulloa.pdf 
Anexo 1 Ejemplo de Actividad

\section{APRENDAMOS A CONTROLAR LOS PROBLEMAS AMBIENTALES DE NUESTRO MUNICIPIO}

\section{EL PROBLEMA DEL PUEBLO DE JATUN}

En un pequeño pueblo llamado Jatun vivían personas trabajadoras, convivían en completa armonía y paz. Su pueblo poseía hermosos y saludables ríos, existía variedad de animales y plantas. Un día, los habitantes decidieron convertir el pueblo de Jatun en un sitio turístico. Entonces construyeron hoteles e hicieron carreteras. Así, los turistas comenzaron a visitar a Jatun.

Los beneficios se empezaron a notar pues todos los negocios crecieron y el dinero aumentaba en el bolsillo de cada habitante. Todo era felicidad cada vez que llegaban los turistas eso implicaba un aumento en sus ingresos. Aumentaba el dinero, aumentaba las ventas, aumentaban las visitas; pero también aumentaba los desperdicios, las basuras, los escombros. Entonces, los habitantes comenzaron a ver sus calles llenas de basuras (residuos sólidos) y de escombros y de erosión causada por la pérdida de los árboles talados en las zonas donde se construyeron los hoteles. Se ocasiono contaminación de aguas, suelo y aire. Entonces los turistas dejaron de llegar y esto llevo a que la tranquilidad y bienestar de este pueblo comenzara a desaparecer.

Pronto los habitantes de la ciudad comenzaron a preguntarsen ¿Qué hacer con todo esto y como solucionar estos problemas?

\section{ACTIVIDAD}

Imagina que tú eres un ciudadano del pueblo de Jatun y que debes crear posibles soluciones para los problemas que se están presentando. Tu participación es muy importante para recuperar la paz y la armonía en este lugar. Para que puedas contribuir de manera responsable a la solución de los problemas que se está presentando en el pueblo de Jatun, sugerimos tener en cuenta lo siguiente:

Lee en voz alta y atentamente el siguiente texto.

Los residuos sólidos son entendidos generalmente como todo material de naturaleza orgánica e inorgánica que ya ha sido utilizado y que muchas veces, se cree que es inservible. Los residuos sólidos son generados en cada una de las actividades que los seres humanos realizamos; a estos les podemos brindar una mejor disposición final, reciclando y clasificándolos adecuadamente.

Los escombros los entendemos como todos los materiales que sobran después de la construcción de cualquier tipo de edificación tales como arenas, gravas, piedra, recebo, asfalto, concreto y agregados sueltos, de construcción o demolición, capa orgánica, suelo y subsuelo de excavación, ladrillo, cemento, acero, hierro, mallas, madera, formaleta y similares que deben ser depositados en lugares apropiados y dispuestos para tal fin. 
Tu profesor será asignado como relator de la actividad, el cual deberá tomar nota de todo lo que suceda, tendrá en cuenta fortalezas, debilidades y el desempeño de cada uno de los participantes.

Una vez, realizado lo anterior te invitamos a que participes en la siguiente actividad. Vamos a simular la ciudad de Jatun dentro del aula de clase, para ello se delegaran a la azar los respectivos roles en el que se representara al alcalde, la secretaria, medico, estudiante de universidad, profesor, ingeniero, policía, obrero, reciclador, empresario, agricultor, aseador, conductor, funcionario de una corporación ambiental, ama de casa y otros.

A continuación te presentamos los principales aspectos que caracterizan a los distintos roles, para que puedas apropiarte de tu personaje:

- Alcalde: Es el rol más importante, ya que este debe escuchar a toda la comunidad, y pensar acciones en beneficio del pueblo

- Secretaria: Apoyo fundamental del alcalde y tomara atenta nota de todo los que digan cada uno de los ciudadanos.

- Medico: Indica todos los posibles perjuicios que podrían presentar a causa de la contaminación tanto a largo como a corto plazo.

- Estudiante de universidad: Mostrará cómo afecta a la universidad y que posible solución se podría buscar con la investigación que desde esta institución educativa se realiza.

- Profesor: Muestra como desde su profesión (muy valiosa para toda la comunidad) realizaría una concienciación a los estudiantes acerca de los problemas.

- Ingeniero: Mostrará como desde su profesión se pueden crear estrategias y sitios propicios para la ubicación de los residuos sólidos y escombros

- Policía: Mostrará como él desde su autoridad podría ayudar a mitigar estos problemas y debe prestar ayuda constante al funcionario de la corporación ambiental.

- Obrero: Revela como desde su labor se generan residuos sólidos y escombros, y la estrategia implementaría para darles un manejo adecuado.

- Reciclador: Desempeña una labor muy importante dentro del manejo de los residuos sólidos y como la ayuda de la comunidad en general le sería de gran ayuda.

- Empresario: Deja ver como la empresa maneja los residuos sólidos y los posibles escombros generados.

- Agricultor: Muestra como se ve afectado por los residuos sólidos que se generan en sus campos en las fuentes de agua y que está haciendo para evitar la generación de erosión.

- Aseador: Demuestra su desagrado por la gran cantidad de residuos sólidos que tiene que recoger cada vez que realiza su labor.

- Conductor: Indica como a él y sus clientes, los escombros que se encuentran en las vías le generan incomodidad cuando se transita por ellas.

- Funcionario de una corporación ambiental: Dice las sanciones y programas que se llevan en contra de la generación de los residuos sólidos y mala disposición de los escombros.

- Ama de casa: Manifiesta si en su casa se realiza algún tipo de manejo de los residuos sólidos. 
Una vez asignado tu rol debes ubicarte junto con tus compañeros en el salón en mesa redonda, y procederás a simular la situación que en Jatun se está presentando, el salón será desordenado con todos los residuos sólidos que los estudiantes trajeron desde sus casas y se simularan los escombros y la erosión.

1. Luego de que te hayas apropiado de tu rol, se comenzara un debate acerca de la situación que se está presentado en Jatun y de la responsabilidad que tiene cada uno (roles) acerca de estos problemas, también todo lo que se podría hacer para buscar las posibles soluciones a estos. Todo esto simulara o recreara un dialogo de saberes (siempre desde cada uno de los roles que tú y tus compañeros tenga a su cargo).

2. Una vez se dé por terminada la dramatización, redacta una conclusión a partir del rol que te fue asignado y una en general de todo lo que se realizó.

3. El relator con todos sus apuntes redactara una conclusión general en donde se evidencie: fortalezas y debilidades, recomendaciones de todo lo visto y la leerá en voz alta.

4. Todos los participantes colaboran en organizar nuevamente el salón. 